\title{
Contagens de ovos por grama de fezes para o controle anti-helmíntico em bovinos de leite de diferentes faixas etárias
}

\author{
Eggs per gram of feces counting for anthelmintic control in dairy cattle of distinct age groups
}

\author{
Ana Maria Antonello ${ }^{I}$ Alfredo Skrebsky CezarII Luís Antônio Sangioni ${ }^{\mathrm{II}}$ \\ Fernanda Silveira Flôres Vogel ${ }^{\mathrm{II} *}$
}

\begin{abstract}
- NOTA -
RESUMO

O controle anti-helmíntico tem vital importância na produtividade de bovinos de leite e, para sua melhor aplicação, devem ser considerados fatores como a sustentabilidade, o custo/benefício e as práticas de manejo utilizadas em cada rebanho. Este estudo foi realizado com o objetivo de estabelecer critérios para o tratamento antihelmíntico de bovinos de leite de categorias distintas com base em contagens de ovos por grama de fezes (OPG). Para isso, analisaram-se amostras fecais de 100 bovinos de leite, em diferentes faixas etárias, de pequenas propriedades da região Centro do Rio Grande do Sul, Brasil. Considerando-se os resultados obtidos neste estudo, são indicados os tratamentos seletivos, com base na contagem de OPG, para as fêmeas jovens (até os três meses de vida) e para as vacas lactantes, e o controle estratégico para os bezerros (dos quatro aos 24 meses).

Palavras-chave: controle estratégico, tratamento seletivo, $O P G$, agricultura familiar.

\section{ABSTRACT}

The control of helminth infections has an essential role in the productivity of dairy cattle. Sustainability, cost/benefit and feasibility of the chosen control measures, must be taking into account for each production system. This study aimed to establish criteria for anthelmintic treatment of dairy cattle, in different categories, based on eggs per gram of feces (EPG) counting. For this purpose, it were analyzed fecal samples from 100 dairy cattle, in different age groups, from small farms of the Central region of Rio Grande do Sul state, Brazil. Based on these results, the selective treatments, based on EPG, are indicated to female calves (until three months old) and lactating

cows, and the strategic control to male and female calves, from four to 24 months old.

Key words: strategic control, selective treatment, EPG, family farming.

As infecções por nematódeos gastrintestinais causam importantes perdas produtivas em bovinos de leite. As nematodíases subclínicas, nas quais há queda no desempenho produtivo sem a manifestação de sinais clínicos, são as mais comuns, havendo ainda os gastos com antiparasitários e os prejuízos com o eventual descarte de leite no póstratamento, durante o período de carência (GROSS et al., 1999; STROMBERG \& GASBARRE, 2006).

As helmintoses estimulam respostas fisiológicas desfavoráveis ao bem-estar e à produtividade dos bovinos; todavia, esse impacto pode ser reduzido por meio de adequadas medidas de controle antiparasitário, envolvendo o manejo do rebanho e as aplicações de anti-helmínticos (ARAÚJO et al., 1992; FOX et al., 2007). Para isso, porém, é necessária uma abordagem epidemiológica dessas infecções, com suas especificidades locais e regionais.

Muitas vezes o controle antiparasitário aplicado nos bovinos não obedece a critérios técnicos, especialmente em propriedades de pequeno porte. Esse
\end{abstract}

ICurso de Medicina Veterinária, Universidade Federal de Santa Maria (UFSM), Santa Maria, RS, Brasil.

IIPrograma de Pós-graduação em Medicina Veterinária, UFSM, Santa Maria, RS, Brasil.

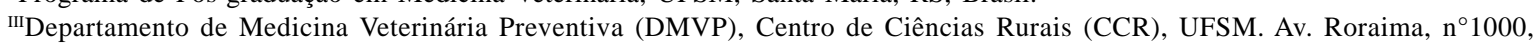
Prédio 44, Camobi, 97105-900, Santa Maria, RS, Brasil. E-mail: fervogel@smail.ufsm.br.*Autor para correspondência. 
fato evidencia a negligência em relação ao conhecimento epidemiológico no direcionamento do controle de helmintos nesses rebanhos. Por isso, neste estudo, avaliou-se o padrão das nematodíases gastrintestinais tomando como base as médias das contagens de ovos por grama de fezes (OPG) em bovinos de leite de faixas etárias distintas, com o objetivo de estabelecer critérios para o tratamento antihelmíntico desses bovinos, com relação às categorias animais nas quais se distribuem.

Para tal, no período entre fevereiro e março de 2008, foram coletadas e analisadas 100 amostras de fezes de bovinos de leite sem raça definida, com idades entre 30 dias e 12 anos, em sete propriedades de pequeno porte, de caráter familiar, localizadas na região Centro do Estado do Rio Grande do Sul, Brasil, na mesorregião do Centro Ocidental Rio-grandense. Utilizaram-se todas as vacas lactantes e todos os bezerros presentes nas propriedades, exceto aqueles que haviam recebido tratamento anti-helmíntico nos 60 dias precedentes à coleta e aquelas fêmeas com crias com idade inferior a 28 dias. Nesses locais, o controle dos helmintos era realizado por meio de tratamentos profiláticos, sem critérios definidos para a avaliação da necessidade e do melhor momento para a aplicação dos antiparasitários. Após os primeiros dias de vida, os bezerros tinham acesso limitado às suas mães, recebendo complementação alimentar com silagens de milho e pastagens cultivadas até o desmame total.

As amostras fecais foram coletadas imediatamente após a ordenha nas vacas lactantes e, em seguida, nos animais jovens. Estas foram identificadas individualmente e acondicionadas sob refrigeração até a realização de contagens de OPG pela técnica de Gordon \& Whitlock - modificada, com margem de detecção de 50OPG (HOFFMANN, 1987). Foram comparados, então, o percentual de animais infectados e o seu grau de infecção nos diferentes grupos etários. Os animais com idade de 0 a 24 meses $(n=53)$ foram reunidos no grupo 1 (G1), enquanto o grupo 2 (G2) foi composto de animais com idade acima de 24 meses $(n=47)$. Para uma análise mais profunda dos dados, o G1 foi subdividido em intervalos de faixa etária: 0 a 3 meses $(n=9) ; 4$ a 7 meses $(n=10) ; 8$ a 12 meses ( $n=13) ; 13$ a 20 meses $(n=11) ; 21$ a 24 meses $(n=10)$.

Os dados de OPG foram analisados depois de submetidos à distribuição normal por meio de transformação logarítmica $(\log X+1,5)$. Foram comparados o G1 e o G2, os subgrupos etários do G1, e estes com o G2. Os percentuais de animais com OPG positivo (prevalência) e de animais com OPG $\geq 300$ para os quais se indicaria o tratamento anti-helmíntico
(HOFFMANN, 1987) - foram analisados pelo teste do Qui-quadrado $(\mathrm{P}<0,05)$, e as médias de OPG foram analisadas pelo teste de Tukey, com significância de $5 \%$.

A distribuição dos intervalos de OPG é demonstrada na figura $1 \mathrm{~A}$. A prevalência foi de 25,5\% nos animais adultos (G2) e de $60,4 \%$ nos animais do G1, com diferença significativa. Dentro do G1, a prevalência foi maior na faixa dos 4 a 7 meses, mantendose, sem diferença significativa, até a faixa dos 13 a 20 meses. Porém, foi mais baixa $(\mathrm{P}<0,05)$ nos animais mais jovens ( 0 a 3 meses de vida), nos animais com 21 a 24 meses e no G2 (adultos). Contudo, sem diferença significativa se comparadas as faixas etárias de 0 a 3 e de 13 a 20 meses. A proporção de animais com OPG=300 (Figura 1A) foi inferior no G2 (10,6\%) em relação ao G1 $(35,8 \%)(P<0,05)$ e não diferiu entre os subgrupos do G1 desde os 0 até os 20 meses de idade, com pico de $60 \%$ na faixa dos 4 a 7 meses. Observa-se, ainda, que não houve diferença significativa entre o subgrupo dos 21 aos 24 meses, no qual esse índice foi zero, e o G2. Além disso, houve equivalência estatística para o subgrupo de 0 a 3 meses e o G2.

Em relação às médias de OPG (Figura 1B), obteve-se média inferior $(\mathrm{P}<0,05)$ no $\mathrm{G} 2(268)$ em relação ao G1 (593). Nos subgrupos do G1, a maior média observada ocorreu na faixa dos 4 a 7 meses, com diferença estatística para os subgrupos de 0 a 3 e de 21 a 24 meses. Entretanto, excetuando-se a faixa etária dos 4 a 7 meses, não houve diferença estatística entre os demais subgrupos do G1.

Esses resultados confirmam que o grau de parasitismo, avaliado pela excreção de ovos nas fezes (OPG), costuma ser maior em bovinos jovens do que em adultos (RIÊT-CORREA et al., 2001; KEYYU et al., 2005). Isso ocorre porque, associada à proteção imunológica prévia do animal, a resposta imune contra os nematódeos do TGI se torna mais eficiente a partir dos 18 a 24 meses de idade. Logo, a partir dessa faixa etária, em geral, os bovinos albergam um número reduzido de parasitas adultos, o que se reflete em uma menor prevalência das infecções no rebanho, além de baixas contagens de OPG e baixa incidência de casos clínicos (CHARLES, 1992). Assim, os menores índices de parasitismo (entre os 20 e os 24 meses) coincidem com um momento em que as novilhas, além de estarem com sua imunidade ativa já estabelecida, ainda não entraram em períodos de gestação e lactação.

Demonstra-se que os bezerros necessitam de tratamentos mais frequentes, podendo ser enquadrados em um programa de controle estratégico, a exemplo daquele proposto por GUIMARÃES et al. (2000), porém levando-se em conta as particularidades 


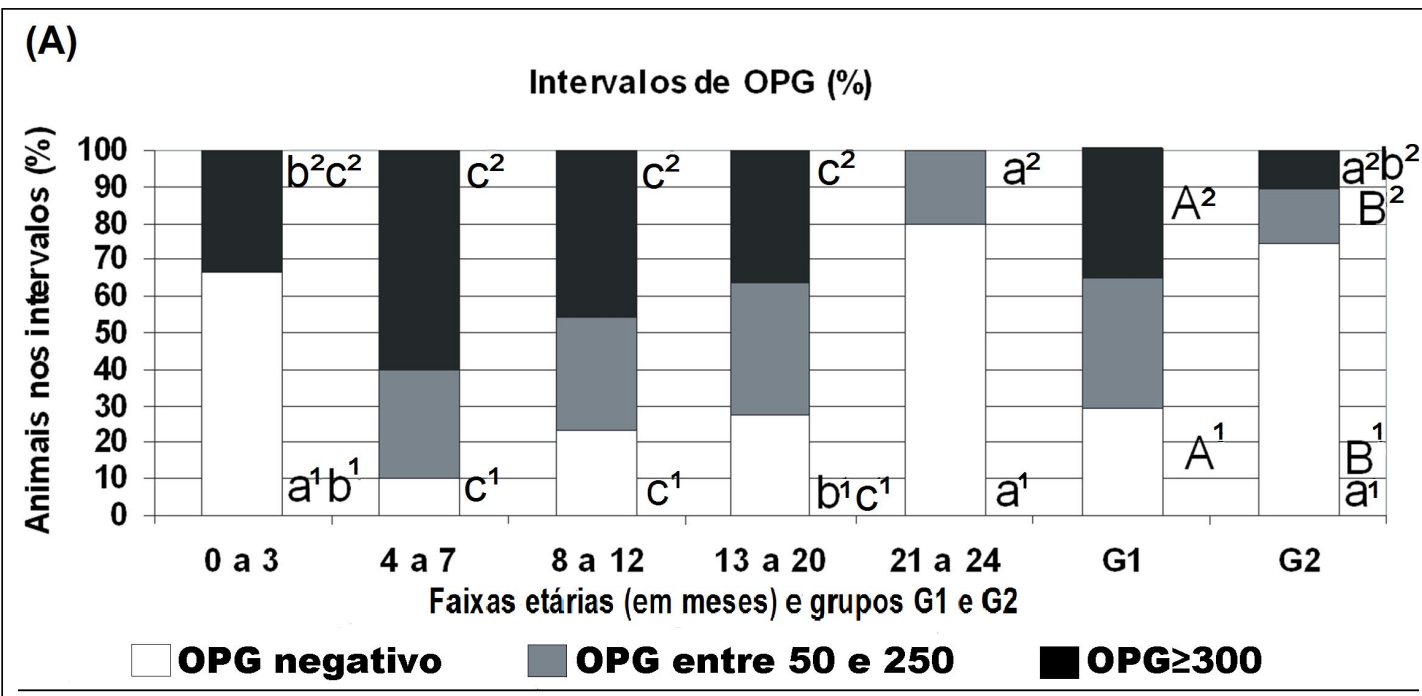

(B)

Médias de OPG

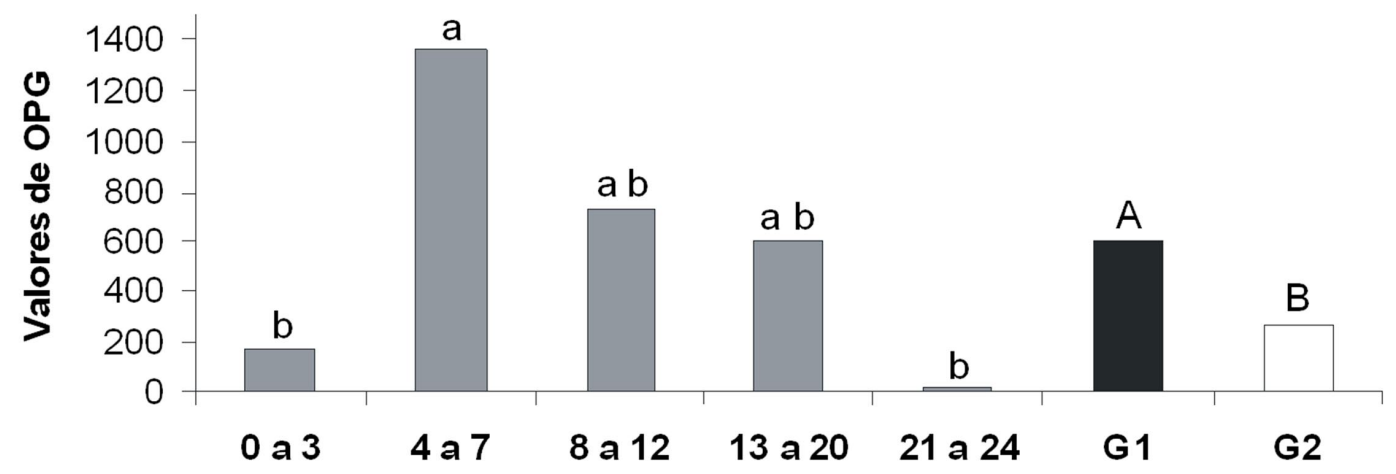

Faixas etárias (em meses)

Grupo 1 (G1)

Grupo 2 (G2)

Figura 1 - Taxas de infecção por nematódeos gastrintestinais em bovinos de leite de diferentes faixas etárias, com base em contagens de ovos por grama de fezes (OPG). G1: animais com idade entre zero e 24 meses. G2: animais com mais de 24 meses. (A) - Distribuição da infecção por nematódeos gastrintestinais, considerando-se o OPG (negativo; entre 50 e 250; e $\geq 300$ ), por faixa etária. (B) - Médias de OPG, por faixa etária. Letras diferentes indicam diferença estatística $(\mathrm{P}<0,05)$ : as minúsculas representam as comparações entre os subgrupos de G1 e destes com G2, e as maiúsculas indicam as comparações entre G1 e G2. ${ }^{1}$ Prevalência (percentual de animais infectados). ${ }^{2}$ Percentual de animais com OPG 300.

regionais da relação ambiente/parasita/hospedeiro. Comparando-se as diferentes faixas etárias do G1, pode-se indicar o primeiro tratamento anti-helmíntico a partir dos quatro meses de vida, quando os animais passam a apresentar alto grau de parasitismo, em razão da maior ingestão de larvas ao pastejo e do decréscimo da resposta imune passiva. No entanto, caso o manejo de desmame dos bezerros seja realizado logo após a ingestão do colostro, incorrendo-se em condições estressantes e induzindo-se precocemente o pastejo, muitas vezes, em um ambiente com alta taxa de contaminação, pode-se considerar a antecipação do tratamento destes animais. Nesse caso, recomenda-se o tratamento seletivo daqueles animais que apresentem contagens de OPG $\geq 300$ (HOFFMANN, 1987), mesmo antes dos quatro meses de vida.

As vacas, pela menor susceptibilidade e, por consequência, pelos menores índices de infecção parasitária, se enquadram em um esquema de controle seletivo, que pode basear-se no monitoramento individual de OPG. Entretanto, vacas que eventualmente passem por condições de estresse, além de sofrerem perdas produtivas, atuarão como fonte de contaminação ambiental, ao excretarem altas taxas de ovos de helmintos nas fezes. Isso se observa, também, no período periparto, especialmente na primeira e na 
segunda gestação (GENNARI et al., 2002). Essas vacas devem ser tratadas, preferencialmente, durante o período seco, podendo-se realizar o tratamento na sua última quinzena de gestação, sendo utilizados antiparasitários aprovados para uso em vacas prenhes. Durante a fase de lactação, o tratamento seletivo dos animais com elevado OPG deve ser realizado com drogas que apresentem curto período de carência para o leite, evitando maiores prejuízos ao produtor.

Conclui-se que o monitoramento do OPG pode ser utilizado como parâmetro para o tratamento seletivo de bovinos de leite, tanto na idade adulta, quanto em animais jovens. Além disso, é importante que sejam privilegiadas estratégias de controle antihelmíntico diferenciadas entre as categorias animais que compõem os rebanhos leiteiros, e que estas sejam baseadas em fatores epidemiológicos. Essas medidas serão benéficas em razão da redução de gastos com tratamentos, de resíduos no leite e no ambiente, da resistência parasitária e de perdas produtivas.

\section{REFERÊNCIAS}

ARAÚJO, J.V. et al. Avaliação de tratamentos anti-helmínticos em bezerros da bacia leiteira de Muriaé, MG. Pesquisa Agropecuária Brasileira, v.27, n.1, p.4-7, 1992. Disponível em: <http://webnotes.sct.embrapa.br/pdf/pab1992/janeiro/ pab02_jan_92.pdf $>$. Acesso em: 25 jun. 2008.

CHARLES, T.P. Verminose dos bovinos de leite. In: CHARLES, T.P.; FURLONG, J. Doenças parasitárias dos bovinos de leite. Coronel Pacheco: Embrapa - CNPGL, 1992. 134p.

FOX, M.T. et al. Epidemiology of subclinical dairy cow nematode infections on five farms in England in 2002 and a comparison with results from 1978 to 1979 . Veterinary Parasitology, v.146, n.3-4, p.294-301, 2007. Disponível em: < h t t p : / / w w w. s c i e n c e d i r e c t. c o m/ science?_ob=ArticleURL\&_udi=B6TD7-4NFRV2N$2 \&$ \&user $=10 \&$ \&doc $=1 \&$ \& fmt $=\&$ \&orig $=$ search $\&$ _sort $=$ d\&_docanchor $=\&$ view $=$ c \&_searchStrId $=1166945488 \&$ rerunOrigin $=$ scholar.google $\&$ _acct $=\mathrm{C} 000050221 \&$ \&version $=1$ \&_urlVersion=0\&_userid=10\&md5=2ed9035b3bc1f4da4f389a8e6a624520>. Acesso em: 25 jun. 2008. doi: 10.1016/j.vetpar.2007.02.020.
GENNARI, S.M. et al. Determinação da contagem de ovos de nematódeos no período peri-parto em vacas. Brazilian Journal of Veterinary Research and Animal Science, v.39, n.1, p.32-37, 2002. Disponível em: <http://www.scielo.br/scielo.php?pid=S141395962002000100006\&script=sci_arttext\&tlng=pt $>$. Acesso em: 6 set. 2008. doi: 10.1590/S1413-95962002000100006.

GROSS, S.J. et al. Anthelmintic treatment of dairy cows and its effect on milk production. Veterinary Record, v.144, n.21, p.581-587, 1999. Disponível em: <http:// veterinaryrecord.bvapublications.com/cgi/content/abstract/144/ 21/581>. Acesso em: 13 mai. 2008.

GUIMARÃES, M.P. et al. Strategic control of gastrointestinal nematodes in dairy calves in Florestal, Minas Gerais, Brazil. Veterinary Research Communications, v.24, n.1, p.3138, 2000. Disponível em: <http://www.springerlink.com/ content/u454j142n5p435t1/fulltext.pdf $>$. Acesso em: 2 jul. 2009. doi: 10.1023/A:1006373221169.

HOFFMANN, R.P. Diagnóstico de parasitismo veterinário. Porto Alegre: Sulina, 1987. 156p.

KEYYU, J.D. et al. Epidemiology of gastrointestinal nematodes in cattle on traditional, small-scale dairy and large-scale dairy farms in Iringa district, Tanzania. Veterinary Parasitology, v.127, n.3-4, p.285-294, 2005. Disponível em: <http:// w w w. s c i e $n$ c e d i r e c t . c o m / science?_ob=MImg\&_imagekey=B6TD7-4F1W6SW-1$3 \&$ _cdi $=5191 \&$ \&user $=687358 \&$ \&orig $=$ search $\&$ _coverDate $=0$ 2\%2F28\%2F2005\&_sk=998729996\&view=c\&wchp=dGLbVlzzSkWz\&md5=8807f8729371a916ec562 aadf4faee96\&ie $=$ / sdarticle.pdf $>$. Acesso em: 26 jun. 2008. doi: 10.1016/ j.vetpar.2004.10.014.

RIET-CORRÊA, F. et al. Doenças de ruminantes e eqüinos. São Paulo: Varela, 2001. 2v. 565p.

STROMBERG, B.E.; GASBARRE, L.C. Gastrointestinal nematode control programs with an emphasis on cattle. Veterinary Clinics of North America: Food Animal Practice, v.22, n.3, p.543-565, 2006. Disponível em: <http:/ / w w w. s c i e $\mathrm{n}$ c e d i r e c t. c o m / science?_ob=MImg\&_imagekey=B7RM9-4M6SC34-6$1 \&$ \&cdi $=25745 \&$ \&_user $=687358 \&$ \&_orig $=$ search \&_cover Date $=11 \% 2 F 30 \% 2 F 2006 \& \_s k=999779996 \&$ view=c\&wchp=dGLbVzWzSkWz\&md5=6a9ea5df4ff204e0cc72fee 78f92e0cf\&ie=/ sdarticle.pdf>. Acesso em: 19 fev. 2009. doi: 10.1016/ j.cvfa.2006.08.003. 